\title{
Effectiveness of Rapid Response Teams on Rates of In-Hospital Cardiopulmonary Arrest and Mortality: A Systematic Review and Meta-analysis
}

\author{
Rose S. Solomon, MPH, Gregory S. Corwin, MPH*, Dawn C. Barclay, MD, MBA, Sarah F. Quddusi, MPH, \\ Michelle D. Dannenberg, MPH
}

The Dartmouth Institute for Health Policy and Clinical Practice, Geisel School of Medicine at Dartmouth, Lebanon, New Hampshire.

BACKGROUND: In 2004, the Institute for Healthcare Improvement's 100,000 Lives Campaign recommended that hospitals implement rapid response teams (RRTs) charged with identifying non-intensive care unit (ICU) patients at risk for rapid deterioration. Although RRTs are now in widespread use, there have been conflicting results regarding the impact of RRTs on hospital mortality and cardiopulmonary arrest.

PURPOSE: To assess the effectiveness of RRTs on reducing hospital mortality and non-ICU cardiopulmonary arrest rates.

DATA SOURCES: We conducted a systematic review using MEDLINE (1966-2014), Cochrane Central Register of Controlled Trials (1898-2014), Cumulative Index to Nursing and Allied Health Literature (1994-2014), and ClinicalTrials.gov (1997-2014) during October 2014. There were no constraints on language or publication status.

DATA EXTRACTION: We included before-after studies, cohort studies, and cluster randomized trials that reported hospital mortality and/or non-ICU cardiopulmonary arrest for adults hospitalized in a non-ICU setting after the implementation of RRTs and/or medical emergency teams (METs). Data were extracted by 2 sets of 2 independent reviewers using a standardized data-collection form. Disagreements were resolved by a third reviewer. Authors were contacted to obtain any missing data.

DATA SYNTHESIS: Our search identified 691 studies, of which 30 met criteria for inclusion in the analysis. Implementation of an RRT/MET was associated with a significant decrease in hospital mortality (relative risk $[R R]=0.88$, $95 \%$ confidence interval $[\mathrm{Cl}]: 0.83-0.93, I^{2}=86 \%$, $3,478,952$ admissions) and a significant decrease in the number of non-ICU cardiac arrests $(\mathrm{RR}=0.62,95 \% \mathrm{Cl}$ : $0.55-0.69, I^{2}=71 \%, 3,045,273$ admissions).

CONCLUSIONS: Implementation of an RRT/MET is associated with a reduction in both hospital mortality and non-ICU cardiopulmonary arrests. Journal of Hospital Medicine 2016;11:438-445. (c) 2016 Society of Hospital Medicine
In 2004, the Institute for Healthcare Improvement (IHI) launched its 100,000 Lives Campaign," a national initiative with a goal of saving 100,000 lives among hospitalized patients through improvements in the safety and effectiveness of healthcare. ${ }^{1}$ One of their recommended strategies to reduce preventable inpatient deaths was for hospitals to establish rapid response teams (RRTs). ${ }^{2,3}$ The goal of RRTs, also termed medical emergency teams (METs), is to identify patients at risk for rapid decline in condition and intervene prior to a catastrophic event such as cardiopulmonary arrest. ${ }^{4}$ The basis for recommending RRT/ METs was evidence of predictable warning signs occurring in patients prior to cardiopulmonary arrest that could alert physicians. ${ }^{5}$ A pilot study by the IHI,

*Address for correspondence and reprint requests: Gregory S. Corwin, VA National Center for Patient Safety Field Office, 215 N. Main St., White River Junction, VT, 05009; Telephone: 802-295-9363; Fax: 802-2916286; E-mail: greg.s.corwin@gmail.com

Additional Supporting Information may be found in the online version of this article.

Received: September 18, 2015; Revised: November 24, 2015; Accepted: January 5, 2016

2016 Society of Hospital Medicine DOI 10.1002/jhm.2554

Published online in Wiley Online Library (Wileyonlinelibrary.com). including 8 hospitals in the United States and the United Kingdom, found reductions in code calls after implementing RRTs, with 2 hospitals also showing a reduction in mortality. ${ }^{3}$

In response to the IHI report, many hospitals established RRT/METs. ${ }^{6}$ Proponents for RRT/METs argued that the potential benefit justified immediate implementation, whereas others advocated for further research. ${ }^{6}$ Despite the rapid, widespread adoption of RRT/METs, questions remain regarding their effectiveness in reducing hospital mortality and non-intensive care unit (ICU) cardiopulmonary arrests. ${ }^{6,7}$ In 2010, Chan et al. reported the results of a meta-analysis of studies published through 2008 that demonstrated a reduction in cardiac arrests, but not mortality, following the implementation of RRTs. ${ }^{8}$ An updated systematic review, including studies published through 2012, suggested that RRTs are associated with reduced non-ICU cardiac arrest and reduced mortality. ${ }^{9}$

Since the publication of the Winters et al. systematic review, several new studies have been published. ${ }^{9-12}$ We performed a systematic review and meta-analysis including studies published through 2014 to examine the impact of RRT/METs on hospital mortality and inhospital cardiopulmonary arrest (IHCA). 


\section{METHODS}

\section{Search Methods}

We conducted a systematic search of publications on RRTs using PubMed (1946-2014), Cumulative Index to Nursing and Allied Health Literature (1937-2014), and the Cochrane Library (issue 10 of 12, 2014). The search used no language restrictions and no limits. Medical Subject Headings with keywords in a Boolean search strategy were employed. The major themes used were cardiopulmonary arrest and rapid response teams. Clinicaltrials.gov (1997-2014) was searched using a similar methodology. A reference review was performed using Web of Science (1900-2014).

\section{Study Eligibility Criteria}

Prespecified criteria for determining study eligibility included: before-after studies, cohort studies, nonrandomized control studies, or cluster randomized controlled trials (RCTs); implementation of an RRT and/or a MET as the intervention; adults (based on individual study definition) hospitalized in a non-ICU setting; reported 1 or both prespecified outcomes, hospital mortality, or IHCA. There were no exclusion criteria or language restrictions.

\section{Data Extraction}

We prospectively outlined a standard protocol that included the research question, inclusion/exclusion criteria, as well as our outcomes and search approaches. We used standard methodology for analysis in accordance with the guidelines in Cochrane Handbook for Systematic Reviews of Interventions. ${ }^{13}$ The protocol can be obtained by request to the authors. All changes to our original protocol were recorded in a protocol amendments table.

The studies identified underwent title and abstract screening by 1 of 2 reviewers (G.S.C., R.S.S.). After irrelevant studies were removed, reviewers independently assessed the remaining studies for eligibility based on full-text review. All disagreements were resolved with consensus and the help of a third reviewer (D.C.B.).

Prior to extracting data, a piloted standardized data-collection form was created. Eligible studies were independently reviewed by each of the 2 reviewers, and the relevant data extracted. Conflicts between the reviewers regarding the data collected for a given study were resolved by a third reviewer. The essential data were total events (hospital deaths and IHCA) and total hospital admissions.

\section{Assessment of Methodological Quality}

We utilized design-specific tools to assess the methodological quality of included studies. For nonrandomized control and cohort studies, we used the Newcastle Ottawa Scale. This allowed us to evaluate the representativeness of the intervention cohort, selection of the nonintervention cohort, ascertainment of the intervention, whether or not the outcome was present at the start of the study, comparability of cohorts, assessment of the outcome, and whether there was adequate follow-up. ${ }^{14}$ We assigned stars as a measure of rating for each category and tallied the number of stars to assess the methodological quality. The maximum score was $9 .{ }^{14}$

For before-after studies, an assessment scale developed by the ECRI (Emergency Care Research Institute) to test the internal validity of each study was utilized. ${ }^{15}$ The ECRI Before-After Scale allowed us to evaluate if the study was prospective, inclusion and exclusion criteria were established a priori, consecutive patients were enrolled, the same initial/subsequent treatment was administered, outcomes were objectively measured, follow-up was complete, cohorts were comparable, there were no conflicts of interest, and conclusions were supported by data. ${ }^{15} \mathrm{We}$ ascertained whether each criterion was met and converted answers to numerical scores. A yes was scored 1, a no was scored -1 , and no response was scored -0.5 . The sum of these scores was then added to 11, divided by 22 , and multiplied by 10 to yield the total quality score. The summary score can range from 0 to 10. A total score $<5$ was considered unacceptable quality. A score $\geq 5$ but $<7.5$ was considered low quality, and a total $\geq 7.5$ was considered moderate quality. ${ }^{15}$

To assess the methodological quality of RCTs, we used the Cochrane Risk of Bias Tool. ${ }^{13}$ The tool involves determining whether a study has a high, low, or unclear risk of bias for specific criteria. ${ }^{13}$

Two independent reviewers evaluated the studies using these scales, and discrepancies were resolved by discussion.

\section{Data Analysis \\ Measure of Treatment Effect}

We used relative risk (RR) to summarize outcome data for our prespecified outcomes: hospital mortality and IHCA.

\section{Dealing With Missing Data}

If essential data were missing, study authors were contacted. If we did not receive a response, we calculated total events (deaths and IHCAs) using total admissions and event rates per admissions. If total admissions and/or event rates were missing, studies were not included in the analysis.

\section{Data Synthesis}

We used Review Manager 5.3 to calculate pooled summary estimates. ${ }^{16}$ Meta-analyses for each outcome were conducted by means of a random effects model.

\section{Assessment of Heterogeneity}

To assess for heterogeneity, we calculated $I^{2}$ and $P$ values. If the $I^{2}<0.50$ or the $P>0.10$, then the test 


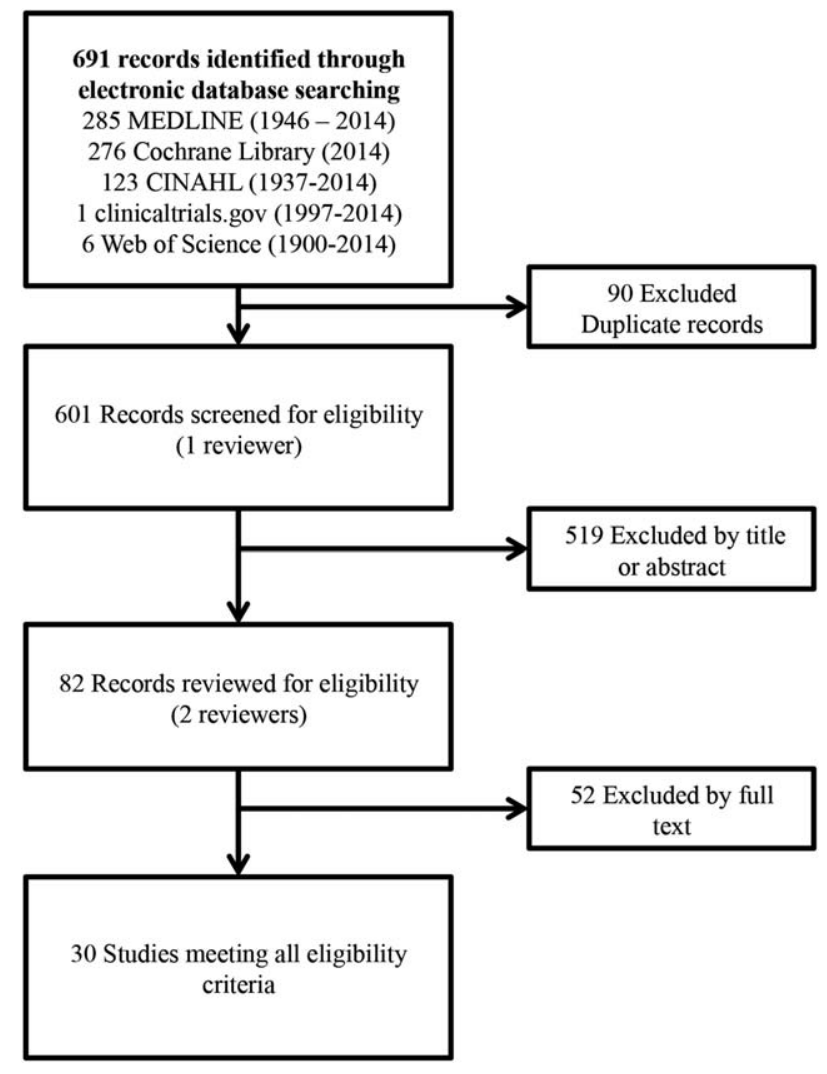

FIG. 1. Study selection flow diagram. Abbreviations: CINAHL, Cumulative Index to Nursing and Allied Health Literature; CENTRAL, Cochrane Central Register of Controlled Trial; MEDLINE = PubMed.

for heterogeneity was passed. If heterogeneity was present, we evaluated each study in an effort to identify outliers. If an outlier was identified, the study was removed from the analysis.

\section{Assessment of Reporting Bias}

To assess publication bias, we used a funnel plot of the primary outcome. The findings were arranged by study size and effect size, and the plot was assessed for symmetry.

\section{Subgroup Analyses}

Subgroup analyses were performed for study type, RRT/MET composition, and publication year. Study type was grouped by cluster RCT and nonrandomized studies versus cohort/before-after studies. Team composition was grouped by whether or not there was a physician on the RRT/MET. Publication year was grouped by studies published before or after 2010 .

\section{Sensitivity Analysis}

We conducted sensitivity analyses to evaluate the impact of methodological quality on summary estimates. We compared overall summary estimates to summary estimates based only on before-after studies judged to be low risk for bias. We also conducted an analysis to evaluate the inclusion of studies in which total events were calculated from rates and total admissions. We compared the overall summary estimates to summary estimates based on studies in which we were able to obtain essential data.

\section{RESULTS \\ Description of Studies}

Our search identified 691 studies, of which 90 were duplicates. The remaining studies were screened by title and abstract, identifying 82 potentially eligible studies, of which 30 studies were identified as eligible for inclusion in the meta-analysis (Figure 1).

Of the 30 eligible studies, 10 were excluded from pooled estimates for hospital mortality, ${ }^{7,17-28}$ and 10 were excluded from pooled estimates for IHCA due to missing data. ${ }^{17,18,20-27,29,30}$ For the analysis, 20 studies were included for the hospital mortality analysis and 20 studies were included for the IHCA analysis. The 22 studies included in either or both analyses spanned the years 2000 to 2014 . The characteristics of the included studies are summarized in Table 1.

\section{Methodological Quality}

The methodological quality of the 4 cohort studies, based on the New Castle Ottawa Scale, was either 8 or 9 stars. Using the ECRI Before-After Scale, the average quality score of the 17 included before-after studies was 8.41 (range, 7.27-9.32). Included beforeafter studies were of moderate quality, with the exception of 1 of lower quality. The cluster RCT had low risk of bias for random sequence generation, allocation concealment, blinding of participants/personnel, and incomplete outcome data; however, it had unclear risk of bias for blinding of outcome assessment, selective reporting, and sources of bias due to lack of reporting. ${ }^{20}$ Overall, the 22 studies included ranged from moderate to good quality.

\section{Effect of RRT on Hospital Mortality}

Of the 20 studies that reported hospital mortality, 9 favored RRT/METs, ${ }^{10,11,30-36} 10$ found no difference with RRT/METs, ${ }^{12,20,22,28,37-42}$ and 1 favored RRT/ METs for surgical patients while favoring usual care (no RRT/MET) for medical patients ${ }^{29}$ (Figure 2a). The pooled analysis demonstrated that implementation of RRT/METs was associated with a significant reduction in hospital mortality $(\mathrm{RR}=0.88,95 \%$ confidence interval [CI]: 0.83-0.93). There was heterogeneity among the contributing studies $\left(I^{2}=86 \%\right)$.

\section{Effect of RRT on IHCA}

Of the 20 studies that reported rates of IHCA, 12 favored RRT/METs $7,10-12,31,32,34-39$ and 8 found no difference with RRT/METs ${ }^{16,19,20,22,28,33,40-42}$ (Figure $2 \mathrm{~b})$. In the pooled analysis, RRT/METs were associated with a significant reduction in IHCA $(\mathrm{RR}=$ 0.62 , 95\% CI: $0.55-0.69)$. There was moderate heterogeneity among the studies $\left(I^{2}=71 \%\right)$. 


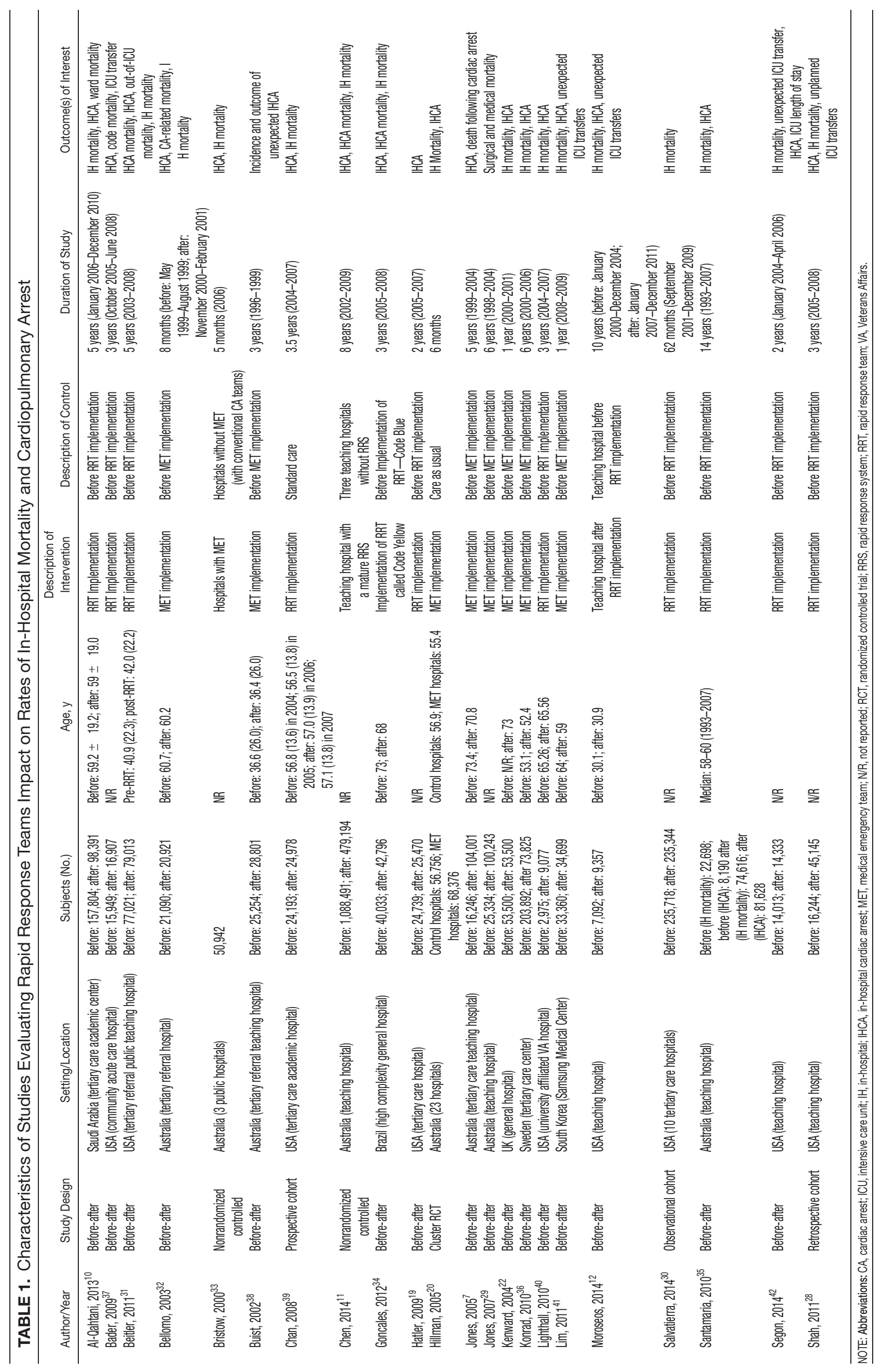




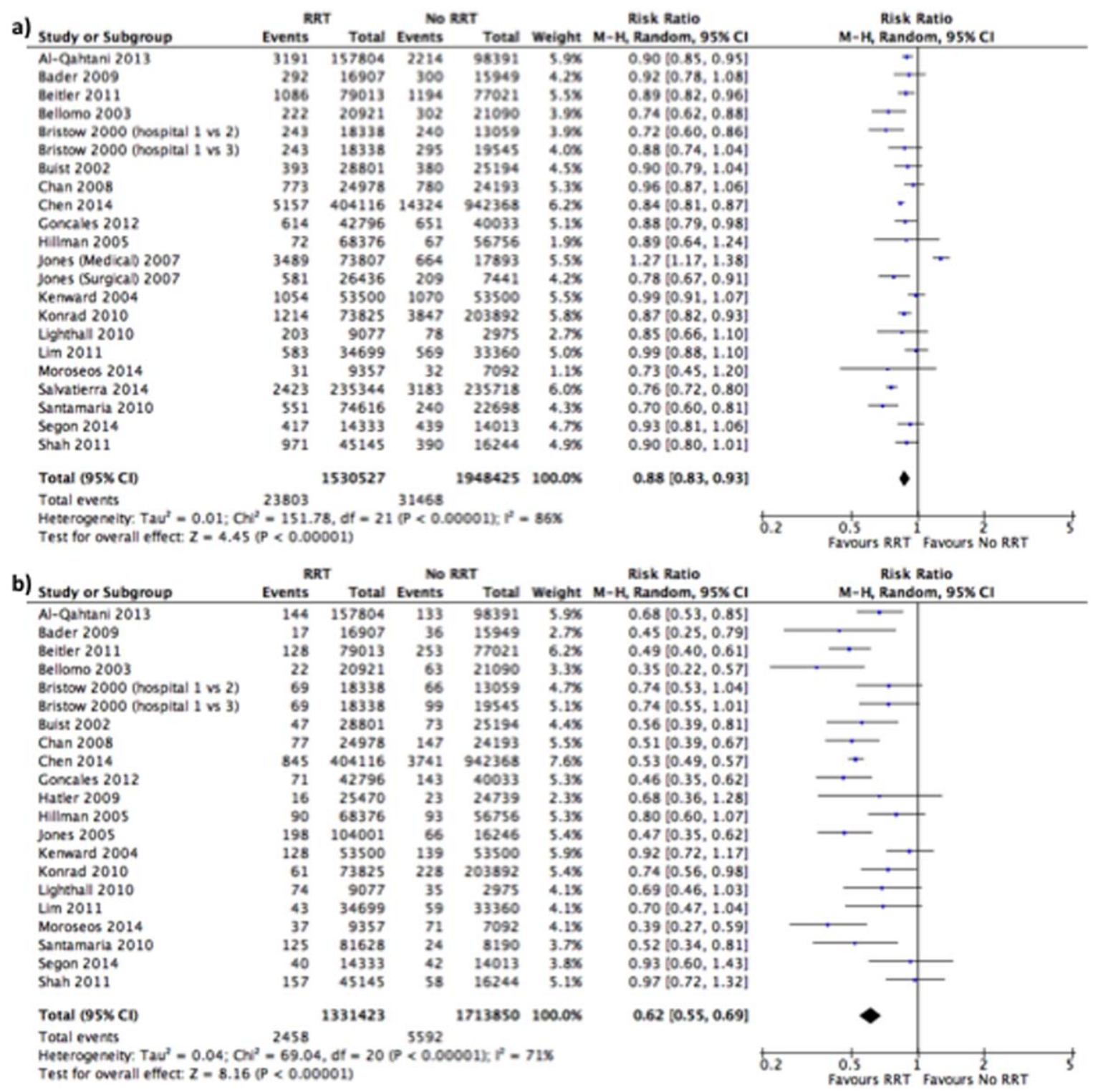

FIG. 2. (a) Forest plot of RRT/MET impact on in-hospital mortality. (b) Forest plot of RRT/MET impact on IHCA. Abbreviations: Cl, confidence interval; $M-H$, Mantel-Haenszel; MET, medical emergency team; RRT, rapid response team.

\section{Subgroup Analysis Study Type}

For hospital mortality, there was 1 cluster RCT and 2 nonrandomized studies ${ }^{11,20,33}(\mathrm{RR}=0.83,95 \%$ CI: 0.80 0.87 ) and 17 cohort/before-after studies ${ }^{10,12,22,28-32,34-42}$ $(\mathrm{RR}=0.89,95 \% \mathrm{CI}: 0.83-0.96)$. The cluster RCT and non-randomized studies had minimal heterogeneity $\left(I^{2}=\right.$ $7 \%)$, and the cohort/before-after studies exhibited substantial heterogeneity $\left(I^{2}=88 \%\right)$. The test for subgroup differences $\left(I^{2}=54.7 \%\right)$ indicates that study type may have an impact on hospital mortality.

For IHCA, there was 1 cluster RCT and 2 nonrandomized studies ${ }^{11,20,33}(\mathrm{RR}=0.68,95 \% \mathrm{CI}$ : 0.52 0.88 ) and 17 before-after studies $7,10,12,19,22,28,31,32,34-42$ $(\mathrm{RR}=0.60,95 \% \mathrm{CI}: 0.52-0.69)$. The cluster RCT and nonrandomized studies had substantial heterogeneity $\left(\mathrm{I}^{2}=79 \%\right)$, whereas the cohort/before-after studies had moderate heterogeneity $\left(I^{2}=69 \%\right)$. The test for sub- group differences $\left(I^{2}=0 \%\right)$ indicates that study type had no impact on IHCA.

\section{RRT/MET Team Composition}

For hospital mortality, there were 14 studies $^{10,20,29,31-38,40-42}$ of RRTs with physicians (RR = 0.88 , 95\% CI: $0.82-0.95)$ and 4 studies ${ }^{12,28,30,39}$ without physicians $(\mathrm{RR}=0.85,95 \% \mathrm{CI}: 0.74-0.99)$. Both groups exhibited substantial heterogeneity $\left(I^{2}=85 \%\right.$ for both). The test for subgroup differences $\left(I^{2}=0 \%\right)$ indicates that team composition had no impact on hospital mortality.

Similarly, for IHCA there were 14 studies $7,10,20,31-38,40-42$ of RRTs with physicians (RR = $0.61,95 \%$ CI: $0.54-0.69)$ and 4 studies ${ }^{12,19,28,39}$ without $(\mathrm{RR}=0.60,95 \%$ CI: 0.39-0.92). The studies with physicians on the RRT had moderate heterogeneity $\left(I^{2}=55 \%\right)$, whereas studies without a physician 
on the RRT had substantial heterogeneity $\left(I^{2}=\right.$ $81 \%)$. The test for subgroup differences $\left(I^{2}=0 \%\right)$ indicates that team composition had no impact on IHCA.

\section{Publication Year}

Publication year had no impact on hospital mortality. Studies published 2010 or earlier had an RR of 0.88 (95\% CI: 0.80-0.97), whereas studies published after 2010 had an RR of 0.87 (95\% CI: 0.83-0.92). Both groups had substantial heterogeneity $\left(I^{2}\right.$ of $88 \%$ and $75 \%$, respectively). The test for subgroup differences $\left(I^{2}=0 \%\right)$ indicates publication year had no impact on hospital mortality.

Publication year had no impact on IHCA. Studies published in 2010 or earlier had an RR of $0.63(95 \%$ CI: 0.54-0.73), whereas studies published after 2010 had an RR of 0.60 (95\% CI: 0.50-0.72). The 2010 or earlier group had moderate heterogeneity $\left(I^{2}=60 \%\right)$, whereas the post-2010 group had substantial heterogeneity $\left(I^{2}=77 \%\right)$. The test for subgroup differences $\left(I^{2}=0 \%\right)$ indicates that publication year had no impact on IHCA.

\section{Sensitivity Analysis}

A sensitivity analysis was performed excluding studies with low methodological quality from the analysis. For hospital mortality there were no studies of low methodological quality. For IHCA there was no major change in the summary estimate or the heterogeneity $\left(\mathrm{RR}=0.59,95 \%\right.$ CI: $\left.0.53-0.67, I^{2}=66 \%\right)$.

A sensitivity analysis was performed excluding studies only reporting rates and/or average annual admissions from the analysis. For hospital mortality, there was no major change in the summary estimate or the heterogeneity $\left(\mathrm{RR}=0.87,95 \%\right.$ CI: $0.82-0.93, I^{2}=$ $87 \%)$. For IHCA there was no major change in the summary estimate, but there was a decrease in heterogeneity (RR $=0.59,95 \%$ CI: $0.53-0.66, I^{2}=63 \%$ ).

\section{Publication Bias}

Funnel plots generated for the effect of RRTs on hospital mortality and on IHCA did not indicate publication bias. Our search of clinicaltrials.gov found 1 potentially eligible study that did not meet our inclusion criteria.

\section{DISCUSSION}

We found implementation of RRT/METs was associated with reductions in hospital mortality and IHCA. Our analysis extends the meta-analysis of Chan et al. and is consistent with the recent systematic review by Winters et al. $^{8,9}$ These findings provide support for the IHI recommendation that hospitals implement RRT/METs. ${ }^{1}$

Following the 2004 IHI recommendations, RRT/ METs were widely implemented, with over $50 \%$ of hospitals having some form of RRT by $2010 .^{6}$ The adoption of RRT/METs occurred despite limited evidence on the effectiveness of RRT/METs. A metaanalysis of studies published through 2008 demonstrated a reduction in cardiac arrests, but no reduction in mortality after implementation of RRT/METs. ${ }^{8}$ More recently a systematic review that included studies through 2012 suggested that RRT/METs are associated with reduced IHCA and reduced mortality. ${ }^{9}$ Our analysis addressed the conflicting results of the prior reviews and included 13 studies published after the Chan et al. meta-analysis and several studies published after the Winters et al. systemic review. ${ }^{8,9}$ The studies included in our analysis were completed in hospitals across multiple countries and settings, increasing the generalizability of the results. Most studies were performed in teaching hospitals; thus, the results may not be as applicable to community hospitals.

We found publication year did not impact either outcome. However, this may reflect our use of 2 broad publication periods rather than smaller periods, as 5 of the 6 newly included studies favor RRT interventions. Additionally, if the studies missing data had been included in our analysis, they may have shown that publication year impacts the outcomes. We noted that a physician on a RRT/MET did not affect outcomes, contrary to suggestions by Winters et al. ${ }^{9}$ This may reflect the skill of nonphysician providers and/or the collaboration of the RRT/MET with critical care teams. However, very few RRTs did not include a physician, limiting the conclusion that can be drawn regarding team composition.

Many patients exhibit observable clinical deterioration or measurable changes that could identify them prior to an event such as cardiac arrest. ${ }^{5,43}$ Measurable physiologic parameters, in fact, are the basis of medical early warning systems and recent automated systems. ${ }^{44,45}$ Similarly, delayed transfer to the ICU has been shown to be associated with increased mortality. ${ }^{46}$ Therefore, RRTs, either by identifying patients at risk for clinical deterioration and/or facilitating transfer of patients to the ICU earlier, could result in improved clinical outcomes. We did not specifically look at ICU transfer or ICU codes in our analysis. However, in a recent single-center before-after study, RRT implementation increased ICU admission rates and the transfer of less severely ill patients to the ICU without improvement in severity of illness-adjusted outcomes. ${ }^{47}$ This finding may reflect the ICU organization of the particular institution; however, given limited ICU resources, admitting an increased number of less severely ill patients without clear clinical benefit is a potential concern. More studies are needed to better understand the mechanism of benefit as well as potential trade-offs associated with RRT implementation. It is possible that institutional factors determine the benefit that can be achieved through RRTs.

Our study has several limitations. Although the methodological quality of the included studies was 
moderate to good, confounding and biases can be an issue with before-after trials and cohort studies. Most studies were before-after observational trials, lacking a concurrent control group making it difficult to draw causal relationships. This is particularly the case for hospital mortality, which has been independently falling since $2000 .^{48}$ Thus, changes in observed hospital mortality may simply reflect the general trend independent of the RRT intervention. However, this does not appear the case for cardiopulmonary arrest, which has been increasing in incidence since $2000 .^{49}$ There were several studies eligible for inclusion in our analysis, but could not be included because of insufficient data. It is possible that the inclusion of these studies could influence the results of our analysis. Finally, there was heterogeneity among the studies for both outcomes, particularly in-hospital mortality. This likely reflects variations in hospital characteristics and case-mix indices. There may also be other factors impacting teams such as how hospitals handled deteriorating patients before RRT implementation, education periods, and differing mechanisms and criteria for RRT activation.

In conclusion, RRT/METs are effective in decreasing both IHCA and hospital mortality. Our findings support the 2004 IHI recommendations for the implementation of RRTs in hospitals. Additional studies are still required to explore team composition, activation criteria, activation mechanism, and implementation strategies.

\section{References}

1. Berwick DM, Calkins DR, McCannon CJ, Hackbarth AD. The 100,000 lives campaign: setting a goal and a deadline for improving health care quality. JAMA. 2006;295(3):324-327.

2. Institute for Healthcare Improvement. Overview of the 100,000 Lives Campaign. Available at: https://www.ihi.org/Engage/Initiatives/ Completed/5MillionLivesCampaign/Documents/Overview\%20of\%20 the \%20100K\%20Campaign.pdf. Accessed September 18, 2014.

3. Whittington J, Simmonds T, Jacobsen D. Reducing Hospital Mortality Rates (Part 2). IHI Innovation Series white paper. Cambridge, MA: Institute for Healthcare Improvement; 2005.

4. Franklin C, Mathew J. Developing strategies to prevent inhospital cardiac arrest: analyzing responses of physicians and nurses in the hours before the event. Crit Care Med. 1994;22(2):244-247.

5. Hillman KM, Bristow PJ, Chey T, et al. Duration of life-threatening antecedents prior to intensive care admission. Intensive Care Med. 2002;28(11):1629-1634.

6. Auerbach AD, Landefeld CS, Shojania KG. The tension between needing to improve care and knowing how to do it. N Engl J Med. 2007; 357(6):608-613.

7. Jones D, Bellomo R, Bates S, et al. Long term effect of a medical emergency team on cardiac arrests in a teaching hospital. Crit Care. 2005; 9(6):R808-R815.

8. Chan PS, Jain R, Nallmothu BK, Berg RA, Sasson C. Rapid response teams: a systematic review and meta-analysis. Arch Intern Med. 2010; 170(1):18-26.

9. Winters BD, Weaver SJ, Pfoh ER, Yang T, Pham JC, Dy SM. Rapidresponse systems as a patient safety strategy: a systematic review. Ann Intern Med. 2013;158(5 pt 2):417-425.

10. Al-Qahtani S, Al-Dorzi HM, Tamim HM, et al. Impact of an intensivist-led multidisciplinary extended rapid response team on hospital-wide cardiopulmonary arrests and mortality. Crit Care Med. 2013;41(2):506-517.

11. Chen J, Ou L, Hillman K, et al. The impact of implementing a rapid response system: a comparison of cardiopulmonary arrests and mortality among four teaching hospitals in Australia. Resuscitation. 2014; 85(9):1275-1281.

12. Moroseos T, Bidwell K, Rui L, et al. Rapid response team implementation on a burn surgery/acute care ward. J Burn Care Res. 2014; $35(1): 21-27$.
13. Higgins J, Green S, eds. Cochrane Handbook for Systematic Reviews of Interventions. Version 5.1.0. Oxford, United Kingdom: The Cochrane Collaboration; 2011: Available at: http://www.cochranehandbook.org. Accessed October 9, 2014.

14. Wells G, Shea B, O'Connell D, et al. The Newcastle-Ottawa Scale (NOS) for Assessing The Quality of Nonrandomised Studies in Metaanalyses. Ottawa, Canada: Ottawa Hospital Research Institute; 2014.

15. Agency for Healthcare Research and Quality. Remote cardiac monitoring: a systematic review. Available at: http://www.cms.gov/determinationprocess/downloads/id51ta.pdf. Published December 12, 2007.

16. Review Manager (RevMan) [computer program]. Version 5.3. Copenhagen, the Netherlands: The Nordic Cochrane Centre, The Cochrane Collaboration; 2014.

17. Bertaut Y, Campbell A, Goodlett D. Implementing a rapid-response team using a nurse-to-nurse consult approach. J Vasc Nurs. 2008; $26(2): 37-42$.

18. Dacey MJ, Mirza ER, Wilcox V, et al. The effect of a rapid response team on major clinical outcome measures in a community hospital. Crit Care Med. 2007;35(9):2076-2082.

19. Hatler C, Mast D, Bedker D, et al. Implementing a rapid response team to decrease emergencies outside the ICU: one hospital's experience. Medsurg Nurs. 2009;18(2):84-90, 126.

20. Hillman K, Chen J, Cretikos M, et al. Introduction of the medical emergency team (MET) system: a cluster-randomised controlled trial. Lancet. 2005;365(9477):2091-2097.

21. Jolley J, Bendyk H, Holaday B, Lombardozzi KA, Harmon C. Rapid response teams: do they make a difference? Dimens Crit Care Nurs. 2007;26(6):253-260; quiz 261-262.

22. Kenward G, Castle N, Hodgetts T, Shaikh L. Evaluation of a medical emergency team one year after implementation. Resuscitation. 2004; 61(3):257-263.

23. Leach LS, Kagawa F, Mayo A, Pugh C. Improving patient safety to reduce preventable deaths: the case of a California safety net hospital. J Healthc Qual. 2012;34(2):64-76.

24. McFarlan SJ, Hensley S. Implementation and outcomes of a rapid response team. J Nurs Care Qual. 2007;22(4):307-313, quiz 314315.

25. Offner PJ, Heit J, Roberts R. Implementation of a rapid response team decreases cardiac arrest outside of the intensive care unit. I Trauma. 2007;62(5):1223-1227; discussion 1227-1228.

26. Priestley G, Watson W, Rashidian A, et al. Introducing Critical Care Outreach: a ward-randomised trial of phased introduction in a general hospital. Intensive Care Med. 2004;30(7):1398-1404.

27. Rothberg MB, Belforti R, Fitzgerald J, Friderici J, Keyes M. Four years' experience with a hospitalist-led medical emergency team: an interrupted time series. J Hosp Med. 2012;7(2):98-103.

28. Shah SK, Cardenas VJ Jr., Kuo YF, Sharma G. Rapid response team in an academic institution: does it make a difference? Chest. 2011; 139(6):1361-1367.

29. Jones D, Opdam H, Egi M, et al. Long-term effect of a medical emergency team on mortality in a teaching hospital. Resuscitation. 2007; $74(2): 235-241$

30. Salvatierra G, Bindler RC, Corbett C, Roll J, Daratha KB. Rapid response team implementation and in-hospital mortality*. Crit Care Med. 2014;42(9):2001-2006.

31. Beitler JR, Link N, Bails DB, Hurdle K, Chong DH. Reduction in hospital-wide mortality after implementation of a rapid response team: a long-term cohort study. Crit Care. 2011;15(6):R269.

32. Bellomo R, Goldsmith D, Uchino S, et al. A prospective before-andafter trial of a medical emergency team. Med J Aust. 2003;179(6): 283-287.

33. Bristow PJ, Hillman KM, Chey T, et al. Rates of in-hospital arrests, deaths and intensive care admissions: the effect of a medical emergency team. Med J Aust. 2000;173(5):236-240.

34. Goncales PD, Polessi JA, Bass LM, et al. Reduced frequency of cardiopulmonary arrests by rapid response teams. Einstein (Sao Paulo). 2012;10(4):442-448.

35. Santamaria J, Tobin A, Holmes J. Changing cardiac arrest and hospital mortality rates through a medical emergency team takes time and constant review. Crit Care Med. 2010;38(2):445-450.

36. Konrad D, Jaderling G, Bell M, Granath F, Ekbom A, Martling CR. Reducing in-hospital cardiac arrests and hospital mortality by introducing a medical emergency team. Intensive Care Med. 2010;36(1): 100-106.

37. Bader MK, Neal B, Johnson L, et al. Rescue me: saving the vulnerable non-ICU patient population. Jt Comm J Qual Patient Saf. 2009;35(4): 199-205.

38. Buist MD, Moore GE, Bernard SA, Waxman BP, Anderson JN, Nguyen TV. Effects of a medical emergency team on reduction of incidence of and mortality from unexpected cardiac arrests in hospital: preliminary study. BMJ. 2002;324(7334):387-390.

39. Chan PS, Khalid A, Longmore LS, Berg RA, Kosiborod M, Spertus JA. Hospital-wide code rates and mortality before and after implementation of a rapid response team. JAMA. 2008;300(21):25062513. 
40. Lighthall GK, Parast LM, Rapoport L, Wagner TH. Introduction of a rapid response system at a United States veterans affairs hospital reduced cardiac arrests. Anesth Analg. 2010;111(3):679-686.

41. Lim SY, Park SY, Park HK, et al. Early impact of medical emergency team implementation in a country with limited medical resources: a before-and-after study. J Crit Care. 2011;26(4):373-378.

42. Segon A, Ahmad S, Segon Y, Kumar V, Friedman H, Ali M. Effect of a rapid response team on patient outcomes in a community-based teaching hospital. J Grad Med Educ. 2014;6(1):61-64.

43. Lighthall GK, Markar S, Hsiung R. Abnormal vital signs are associated with an increased risk for critical events in US veteran inpatients. Resuscitation. 2009;80(11):1264-1269.

44. Kollef MH, Chen Y, Heard K, et al. A randomized trial of real-time automated clinical deterioration alerts sent to a rapid response team. J Hosp Med. 2014;9(7):424-429.

45. Escobar GJ, LaGuardia JC, Turk BJ, Ragins A, Kipnis P, Draper D. Early detection of impending physiologic deterioration among patients who are not in intensive care: development of predictive models using data from an automated electronic medical record. $J$ Hosp Med. 2012;7(5):388-395.

46. Liu V, Kipnis P, Rizk NW, Escobar GJ. Adverse outcomes associated with delayed intensive care unit transfers in an integrated healthcare system. J Hosp Med. 2012;7(3):224-230.

47. Karpman C, Keegan MT, Jensen JB, Bauer PR, Brown DR, Afessa B. The impact of rapid response team on outcome of patients transferred from the ward to the ICU: a single-center study. Crit Care Med. 2013; 41(10):2284-2291.

48. Hall MJ, Levant S, DeFrances CJ. Trends in inpatient hospital deaths: National Hospital Discharge Survey, 2000-2010. NCHS Data Brief. 2013(118):1-8.

49. Kazaure HS, Roman SA, Sosa JA. Epidemiology and outcomes of inhospital cardiopulmonary resuscitation in the United States, 20002009. Resuscitation. 2013;84(9):1255-1260. 